\title{
The MPGD-based photon detectors for the upgrade of COMPASS RICH-1 and beyond
}

\author{
J. Agarwala ${ }^{\mathrm{a}, 1}$, M. Alexeev ${ }^{\mathrm{b}}$, C.D.R. Azevedo ${ }^{\mathrm{c}}, \mathrm{F}$. Bradamante ${ }^{\mathrm{d}, \mathrm{a}}, \mathrm{A}^{\mathrm{A}}$. Bressan ${ }^{\mathrm{d}, \mathrm{a}}$, M. Büchele ${ }^{\mathrm{e}}$, \\ M. Chiosso ${ }^{\text {f,b }}$, C. Chatterjee ${ }^{\mathrm{d}, \mathrm{a}}$, A. Cicuttin ${ }^{\mathrm{a}, 1}$, P. Ciliberti ${ }^{\mathrm{d}, \mathrm{a}}$, M.L. Crespo ${ }^{\mathrm{a}, 1}$, S. Dalla Torre ${ }^{\mathrm{a}, *}$, \\ S. Dasgupta ${ }^{a}$, O. Denisov ${ }^{\text {b }}$, M. Finger ${ }^{\text {g,h }}$, M. Finger Jr. ${ }^{\text {g,h }}$, H. Fischer ${ }^{\mathrm{e}}$, M. Gregori ${ }^{\mathrm{a}}$, G. Hamar ${ }^{\mathrm{a}}$, \\ F. Herrmann ${ }^{\mathrm{e}}$, S. Levorato ${ }^{\mathrm{a}}$, A. Martin ${ }^{\mathrm{d}, \mathrm{a}}$, G. Menon ${ }^{\mathrm{a}}$, D. Panzieri ${ }^{\mathrm{i}, \mathrm{b}}$, G. Sbrizzai ${ }^{\mathrm{d}, \mathrm{a}}$, \\ S. Schopferer ${ }^{\mathrm{e}}$, M. Slunecka ${ }^{\mathrm{g}, \mathrm{h}}$, M. Sulc ${ }^{\mathrm{j}}$, F. Tessarotto ${ }^{\mathrm{a}}$, J.F.C.A. Veloso ${ }^{\mathrm{c}}$, Y. Zhao $^{\mathrm{a}}$
}

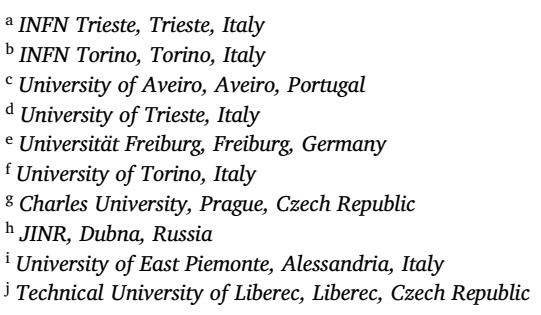

\section{A R T I C L E I N F O}

\section{Keywords:}

Gaseous photon detectors

MPGD

Thick GEM

Resistive MICROMEGAS

COMPASS

\begin{abstract}
A B S T R A C T
After pioneering gaseous detectors of single photon for RICH applications using CsI solid state photocathodes in MWPCs within the RD26 collaboration and by the constructions for the RICH detector of the COMPASS experiment at CERN SPS, in 2016 we have upgraded COMPASS RICH by novel gaseous photon detectors based on MPGD technology. Four novel photon detectors, covering a total active area of $1.5 \mathrm{~m}^{2}$ have been installed in order to cope with the challenging efficiency and stability requirements of the COMPASS physics programme. They are the first application in an experiment of MPGD-based single photon detectors. All aspects of the upgrade are presented, including engineering, mass production, quality assessment and performance.

Perspectives for further developments in the field of gaseous single photon detectors are also presented.
\end{abstract}

Contents

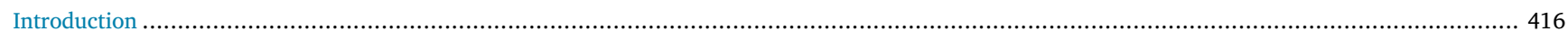

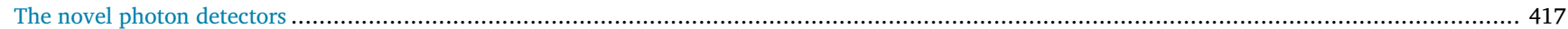

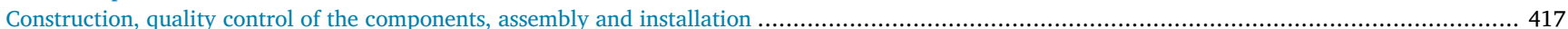

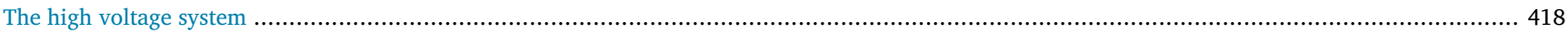

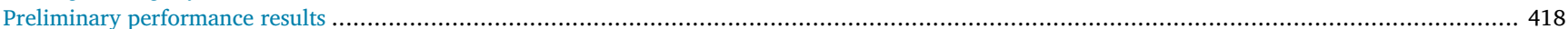

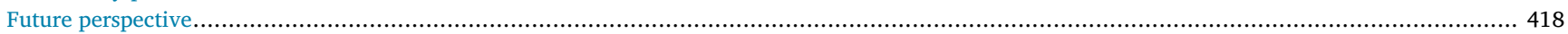

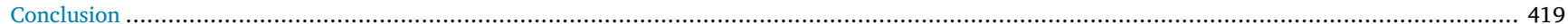

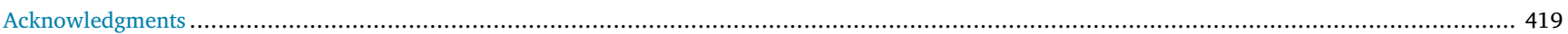

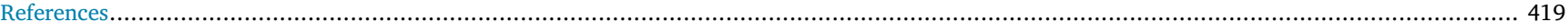

\section{Introduction}

THE RICH-1 detector [1] of the COMPASS Experiment [2] at CERN SPS has been upgraded: four new Photon Detectors (unit size:

\footnotetext{
* Corresponding author.

E-mail address: silvia.dallatorre@ts.infn.it (S.D. Torre).

1 Also at Abdus Salam ICTP, Trieste, Italy.
} 
$600 \times 600 \mathrm{~mm}^{2}$ ), based on MPGD technology and covering a total active area of $1.5 \mathrm{~m}^{2}$ replace the previously used MWPC-based photon detectors in order to cope with the challenging efficiency and stability requirements of the new COMPASS measurements. In fact, COMPASS goal is to deal with trigger rates up to $\mathrm{O}\left(10^{5}\right) \mathrm{Hz}$ and beam rates up to $\mathrm{O}\left(10^{8}\right) \mathrm{Hz}$. Concerning increased stability, this is provided by the new detector architecture, as explained in the following. In COMPASS RICH-1, MPGD photon detectors are used for the first time in a running experiment. This realization also opens the way of a more extended use of novel gaseous photon detectors in the domain of the Cherenkov imaging technique for Particle IDentification (PID), key detectors in several research sectors and, in particular, in hadron physics. The relevance is related to the role of gaseous photon detectors, which are still the only available option to instrument detection surfaces when insensitivity to magnetic field, low material budget, and affordable costs in view of large detection systems are required. The MPGD-based photon detectors overcome the limitation of the previous generation of gaseous photon detectors thanks to two essential performance characteristics: reduced ion and photon backflow to the photocathode, namely reduced ageing and increased electrical stability, and faster signal development, namely higher rate capabilities.

\section{The novel photon detectors}

The detector architecture is the result of a seven-year R\&D activity [3]. It is based on a hybrid MPGD combination (Fig. 1), consisting in two layers of THick GEMs (THGEM) [4] followed by a resistive MicroMegas (MM) [5] on a pad segmented anode. The first THGEM also acts as a reflective photocathode: its top face is coated with a CsI film. The feedback of photons generated in the multiplication process is suppressed by the presence of two THGEM layers, while the large majority of the ions from multiplication are trapped in the MM stage. MPGD properties ensure signal development in about $100 \mathrm{~ns}$.

Each of the four large $\left(600 \times 600 \mathrm{~mm}^{2}\right)$ single photon detectors is formed by two identical modules $600 \times 300 \mathrm{~mm}^{2}$, arranged side by side. The THGEM geometrical parameters are: $470 \mu \mathrm{m}$ thickness, $400 \mu \mathrm{m}$ hole diameter and $800 \mu \mathrm{m}$ pitch. Holes are rim-less, namely there is no uncoated area around the hole edge. They are arranged in a regular pattern with equilateral triangles as elementary cell. In order to mitigate the effect of occasional discharges, the top and bottom electrodes of each THGEM are segmented in 12 parallel areas separated by $0.7 \mathrm{~mm}$ clearance, each biased via an individual protection $500 \mathrm{M} \Omega$ resistor. Therefore, discharges only affect a single sector and the operating conditions are restored in about $10 \mathrm{~s}$. The two layers are staggered, namely there is complete misalignment between the two set of holes: it is so possible to enlarge the electron cloud reaching the MM stage, therefore favoring larger gain in the last amplification stage.

The MMs have a gap of $128 \mu \mathrm{m}$; they are built by the MM bulk technology [6] using $300 \mu \mathrm{m}$ diameter pillars with $2 \mathrm{~mm}$ pitch. The MM anode is segmented in $7.5 \times 7.5 \mathrm{~mm}^{2}$ pads. The MM resistivity is realized through an original implementation, where no resistive layer is applied to the pads: the resistivity is obtained by $470 \mathrm{M} \Omega$ resistors in series with each individual pad (Fig. 2). The $0.5 \mathrm{~mm}$ clearance between pads prevents the occasional discharges to propagate towards the surrounding pads: the voltage drop of the anodic pads surrounding a tripping one is about $2 \mathrm{~V}$ over the typical $600 \mathrm{~V}$ operation voltage, causing a local gain drop lower than $4 \%$. The nominal voltage condition of the pad where the discharge occurred is restored in about $1 \mathrm{~s}$. The detector is operated with $\mathrm{Ar}: \mathrm{CH}_{4}=50: 50$ gas mixture, which ensures effective extraction of photoelectrons from the photocathode. The typical voltage applied are $1270 \mathrm{~V}$ across THGEM1, $1250 \mathrm{~V}$ across THGEM2, and $620 \mathrm{~V}$ to bias the MM. The drift field above the first THGEM is $500 \mathrm{~V} / \mathrm{cm}$, the transfer field between the two THGEMs is $1000 \mathrm{~V} / \mathrm{cm}$ and the field between the second THGEM and the MM micromesh is $1000 \mathrm{~V} / \mathrm{cm}$. The effective gain-values for the three multiplication layers are around 12, 10 and 120; these values include the electron transfer efficiency.

The novel detectors are read out by the read-out system already used for the MWPCs with CsI photocathode. This read-out system is based on the APV front-end chip [7] read out by a dedicated ADC [8].

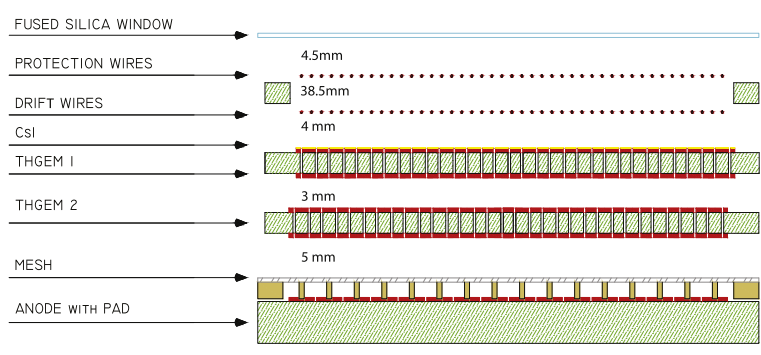

Fig. 1. Sketch of the hybrid single photon detector: two staggered THGEM layers are coupled to a resistive bulk MM. Image not to scale.

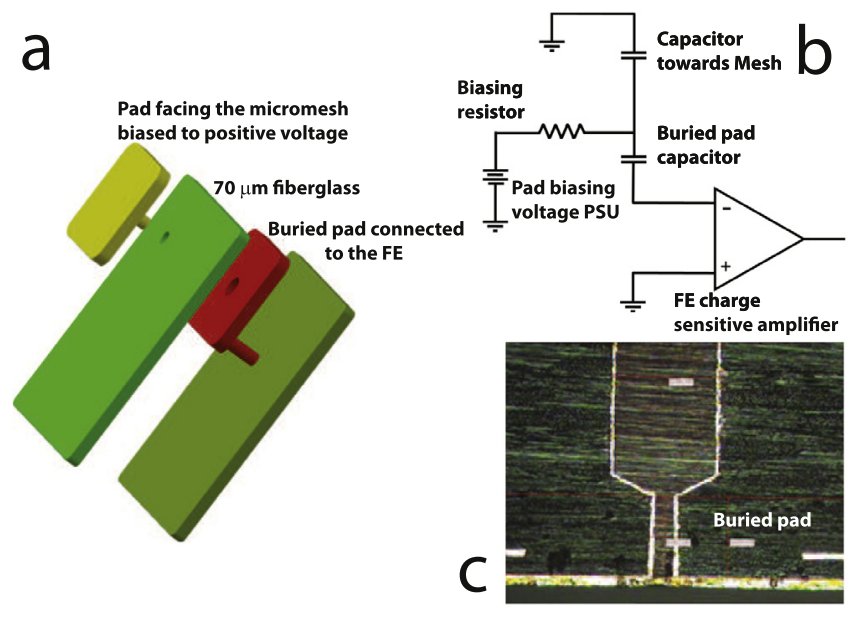

Fig. 2. (a) Sketch of the capacitive coupled readout pad. The biasing voltage is distributed via independent $470 \mathrm{M} \Omega$ resistors to the pad facing the micromesh structure (yellow pad in the sketch). The buried pad (red pad in the sketch) is isolated via $70 \mu \mathrm{m}$ thick fiberglass and connected to the front end chip. (b) Schematic of the capacitive coupled pad principle illustrated via discrete element blocks. (c) Metallography section of the PCB: detail of the through-via connecting the external pad through the hole of the buried pad. The reduced diameter of the through-via reaching the external pad contributes preserving the pad planarity.

3. Construction, quality control of the components, assembly and installation

The electrical stability of large-size THGEMs is a critical issue. A dedicated protocol has been elaborated for finishing the industrial produced THGEMs [9]. It includes polishing with fine grain pumice powder, cleaning with water at high pressure, ultrasonic bath with Sonica PCB solution (PH11), rinsing with distilled water and backing in oven at $160{ }^{\circ} \mathrm{C}$. The procedure moves THGEM breakdown voltage to at least $90 \%$ of the phenomenological Paschen limit [10]. The quality control of the detector components includes:

- the preselection of the raw material for the PCB that will form the THGEMs in order to use only foils with homogeneous thickness to guarantee the homogeneity of the gain;

- the THGEM control by optical inspection, by collecting and analyzing microscope images, scanning by samples the large multiplier surface;

- the THGEM validation by gain maps using the multipliers in single layer detectors; gain uniformity at $7 \%$ r.m.s. is obtained;

- the collection of MM gain maps illuminating the detectors by an $\mathrm{X}$-ray gun station; gain uniformity at $5 \%$ r.m.s. is obtained;

- the measurement of the quantum efficiency of the CsI photocathodes, which is performed immediately after the coating process; the uniformity within a photocathode is at the $3 \%$ level r.m.s. and among the whole production at the $10 \%$ level r.m.s.;

- the gas leak checks and overall electrical stability checks of the final detectors. 
CsI photocathodes must never be exposed to air to fully preserve their quantum efficiency: in fact, CsI is highly hygroscopic and it reacts with water vapour that decomposes the molecule. Therefore, the presence of CsI photocathodes imposes to perform detector assembly, transportation and installation in glove boxes flushed with $\mathrm{N}_{2}$.

\section{The high voltage system}

An essential tool for the detector commissioning is the High Voltage (HV) control system, which also allows for voltage and current monitoring and data logging. The power supplies are commercial ones by CAEN inserted in a SY4527 mainframe. A1561HDN units power the THGEMs, while A7030DP power supplies are used for the MMs. The four detectors are organized, from the HV supply point of view, in four independent sectors each; nine different electrode types, each one with its specific role, are present in the multilayer detectors. The total number of HV channels is 136. Manual setting and control of all these HV channels would be both unpractical and unsafe. The voltages and currents of all the channels are read-out and recorded at $1 \mathrm{~Hz}$ frequency. If the current spark rate is above a given value the voltage is automatically readjusted. The system also provides automatic voltage adjustment to compensate for the variation of the environmental parameters, namely pressure and temperature, that can affect the detector gain. Gain stability at the 5\% level over months of operation has been obtained.

\section{Preliminary performance results}

The novel detectors have been used during COMPASS runs in year 2016 and 2017, for a total running period of about 12 months at COMPASS nominal beam rates. No HV trip is observed during detector operation: thanks to the resistors protecting the THGEM segments and the MM pads, in case of occasional discharges, only current sparks are observed, which temporary affect the local performance. The sparks in the two THGEM layers are fully correlated. The sparks observed in the MM are induced by the THGEM sparks. The restoration after a current spark is completed within $10 \mathrm{~s}$ and the current spark rate is typically $1 / \mathrm{h} /$ detector $\left(600 \times 600 \mathrm{~mm}^{2}\right)$. These figures result in totally negligible dead-time related to sparks.

The measured rate of ion backflow to the photocathode is at the $3 \%$ level. The electronics noise, substantially uniform over the detector surface, is at the 900 electrons equivalent level (r.m.s).

The images generated in the photon detectors are clean and affected by very limited background (Fig. 3). The detector resolution in the measurement of the Cherenkov angle from single photoelectrons is 1.7$1.8 \mathrm{mrad}$ r.m.s., fully matching the expectation (Fig. 4). The amplitude spectrum of the photoelectron signals is expected to be exponential. This is verified for pure photoelectron samples, obtained selecting hits contributing to ring images: the exponential behavior is present over more than two orders of magnitude (Fig. 5). The detector gain is extracted from a fit of the spectrum and it ranges between 13k and $14 \mathrm{k}$. An electronic threshold of 3 times the noise level as measured pad by pad is applied to each read-out channel. The efficiency for single photoelectron detection is obtained from the gain and the threshold and it results higher than $80 \%$. The noise contributing to the ring images can be estimated from the spectrum deviation from a pure exponential function at small amplitude and it is at the $10 \%$ level. A preliminary estimate of the number of detected photoelectrons per particle extrapolated to the saturation angle indicates 11 photoelectrons.

The high effective gain, the gain stability and the number of detected photoelectrons per ring satisfy all the prerequisite requirements to ensure effective hadron identification and stable performance with the novel RICH-1 photon detectors.

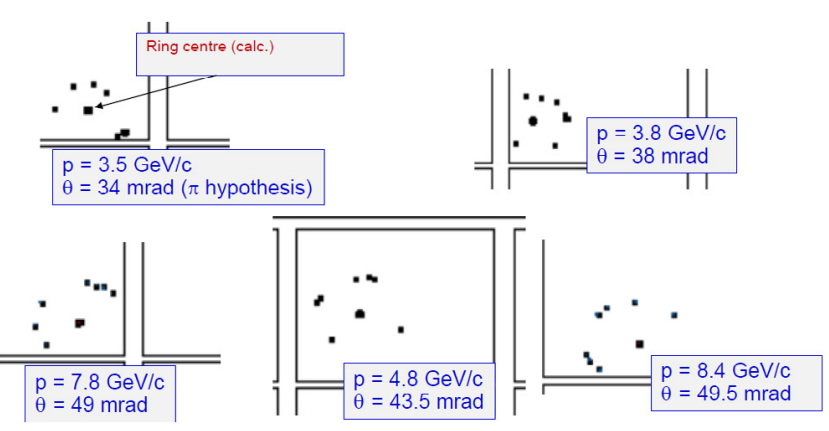

Fig. 3. Images of hit pattern in the novel photon detectors. The center of the expected ring patterns is obtained from the reconstructed particle trajectories; the particle momentum and the expected Cherenkov angle in the pion hypothesis are also reported. No image elaboration or background subtraction is applied.

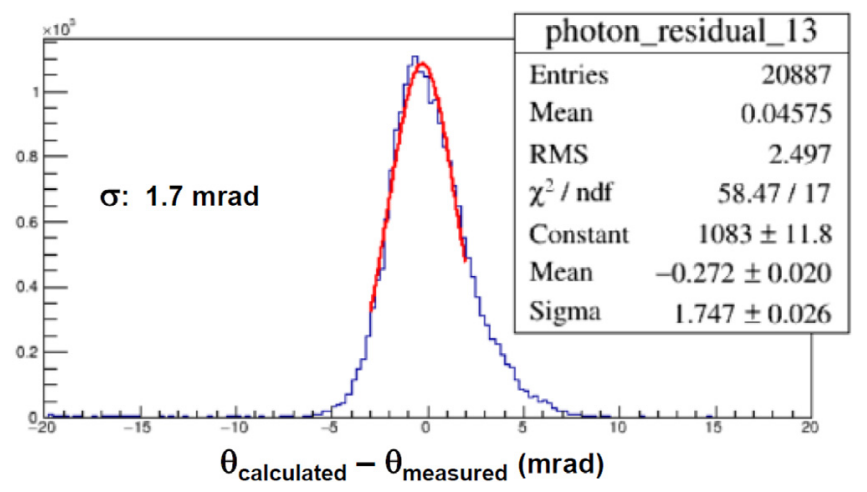

Fig. 4. Distribution of the difference between the Cherenkov angle calculated from the reconstructed particle momentum and the Cherenkov angle provided by single detected photoelectrons; a sample of identified pions is used.

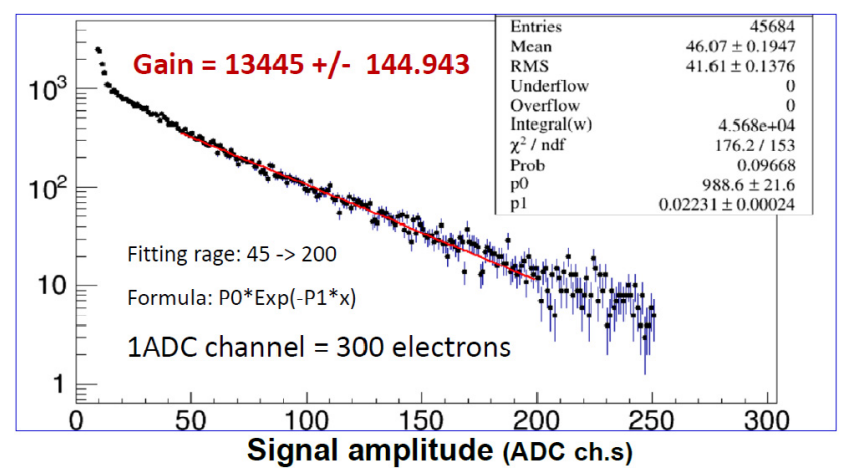

Fig. 5. Amplitude distribution for a sample of hits contributing to ring Cherenkov images.

\section{Future perspective}

The future Electron-Ion Collider (EIC) [11] requires hadron identification at high momenta, a mission that can only be accomplished by RICH counters with an extended gas radiator. The use of RICHes in the setup of collider experiments implies specific challenges. The radiator cannot be too extended to limit the overall apparatus size, imposing the need to detect more photoelectrons per radiator unit length. The photon detectors have to operate in presence of magnetic field. A recent test-beam exercise has demonstrated the possibility to increase the number of detected photoelectrons by selecting the far UV range around $120 \mathrm{~nm}$ [12]. For this purpose, the RICH prototype has been operated window-less and $\mathrm{CF}_{4}$ has been used at the same time as radiator gas and detector gas. Therefore, we have started an R\&D program to match these specific requirements. It includes the exploratory study of a new 
option for the photoconverter: coating by hydrogenized nanodiamond powder [13].

\section{Conclusion}

The preliminary results obtained in the characterization of novel MPGD-based photon detectors, in particular high effective gain, gain stability and number of photons per ring, indicate that they will fully accomplish the mission of increasing the stability and efficiency of the photon detector system of COMPASS RICH-1. They also represent a technological achievement. In fact, for the first time in a running experiment, THGEMs are successfully used, single photon detection is accomplished by MPGDs, MPGDs are operated at gains larger than 10k.

We have offered indications that MPGD-based photon detectors have a mission to accomplish also in the future, in particular in the hadron physics sector.

\section{Acknowledgments}

The authors are grateful to the colleagues of the COMPASS Collaboration for continuous support and encouragement.

This work is partially supported by the H2020 project AIDA-2020, GA no. 654168.

\section{References}

[1] E. Albrecht, et al., Status and characterisation of COMPASS RICH-1, Nucl. Instrum. Methods A 553 (2005) 215;

P. Abbon, et al., Read-out electronics for fast photon detection with COMPASS RICH-1, Nucl. Instrum. Meth. A587 (2008) 371;

P. Abbon, et al., Design and construction of the fast photon detection system for COMPASS RICH-1, Nucl. Instrum. Meth. A616 (2010) 21;

P. Abbon, et al., Particle identification with COMPASS RICH-1, Nucl. Instrum. Meth. A631 (2011) 26.

[2] The COMPASS Collaboration, P. Abbon, et al., The COMPASS experiment at CERN, Nucl. Instrum. Methods A 577 (2007) 455;

COMPASS Collaboration, P. Abbon, et al., The COMPASS setup for physics with hadron beams, Nucl. Instrum. Meth. A779 (2015) 69.

[3] M. Alexeev, et al., The quest for a third generation of gaseous photon detectors for Cherenkov imaging counters, Nucl. Instrum. Methods A 610 (2009) 174;

M. Alexeev, et al., THGEM based photon detector for Cherenkov imaging applications, Nucl. Instrum. Meth. A617 (2010) 396;

M. Alexeev, et al., Micropattern gaseous photon detectors for Cherenkov imaging counters, Nucl. Instrum. Meth. A623 (2010) 129;

M. Alexeev, et al., Development of THGEM-based photon detectors for Cherenkov Imaging Counters, JINST 5 (2010) P03009;

M. Alexeev, et al., Progress towards a THGEM-based detector of single photons, Nucl. Instrum. Meth. A639 (2011) 130;
M. Alexeev, et al., Detection of single photons with ThickGEM-based counters, JINST 7 (2012) C02014;

M. Alexeev, et al., Detection of single photons with THickGEM-based counters, Nucl. Instrum. Meth. A695 (2012) 159;

M. Alexeev, et al., Development of THGEM-based Photon Detectors for COMPASS RICH-1, Phys. Procedia 37 (2012) 781;

M. Alexeev, et al., THGEM-based photon detectors for the upgrade of COMPASS RICH-1, Nucl. Instrum. Meth. A732 (2013) 264;

M. Alexeev, et al., Ion backflow in thick GEM-based detectors of single photons, JINST 8 (2013) P01021;

M. Alexeev, et al., Status and progress of novel photon detectors based on THGEM and hybrid MPGD architectures, JINST 8 (2013) C12005;

M. Alexeev, et al., Progresses in the production of large-size THGEM boards, JINST 9 (2014) C03046;

M. Alexeev, et al., MPGD-based counters of single photons developed for COMPASS RICH-1, JINST 9 (2014) C09017;

M. Alexeev, et al., Status and progress of the novel photon detectors based on THGEM and hybrid MPGD architectures, Nucl. Instrum. Meth. A766 (2014) 133;

M. Alexeev, et al., MPGD-based counters of single photons for Cherenkov imaging counters, PoS (2014) 075;

M. Alexeev, et al., The gain in Thick GEM multipliers and its time-evolution, JINST 10 (2015) P03026;

M. Alexeev, et al., Status of the development of large area photon detectors based on THGEMs and hybrid MPGD architectures for Cherenkov imaging applications, Nucl. Instrum. Meth. A824 (2016) 139.

[4] L. Periale, et al., Detection of the primary scintillation light from dense Ar, Kr and Xe with novel photosensitive gaseous detectors, Nucl. Instrum. Methods A 478 (2002) 377; P. Jeanneret, Time Projection Chambers and Detection of Neutrinos, (Ph.D. thesis), Neuchatel University, 2001.

P.S. Barbeau, et al., Toward coherent neutrino detection using low-background micropattern gas detectors, IEEE NS- 50 (2003) 1285;

R. Chechik, et al., Thick GEM-like hole multipliers: properties and possible applications, Nucl. Instrum. Meth. A535 (2004) 303.

[5] Y. Giomataris, et al., MICROMEGAS: a high-granularity position-sensitive gaseous detector for high particle-flux environments, Nucl. Instrum. Methods A 376 (1996) 29.

[6] I. Giomataris, et al., Micromegas in a bulk, Nucl. Instrum. Methods A 560 (2006) 405.

[7] M.J. French, et al., Nucl. Instrum. Meth. A 466 (2001) 359.

[8] P. Abbon, et al., Nucl, Instrum. Meth. A 589 (2008) 362.

[9] S.S. Dasgupta, Particle identification with the Cherenkov imaging technique using MPGD based photon detectors for physics at COMPASS Experiment at CERN, University of Trieste, 2017.

[10] F. Paschen, Über die zum Funkenübergang in Luft, Wasserstoff und Kohlensäure bei verschiedenen Drucken erforderliche Potentialdifferenz, Ann. Phys. 273 (1889) 69.

[11] A. Accardi, et al., Electron-Ion Collider: The next QCD frontier, Eur. Phys. J. A52 (2016) 268.

[12] M. Blatnik, et al., Performance of a Quintuple-GEM Based RICH detector prototype, IEEE NS 62 (2015) 3256.

[13] L. Velardi, A. Valentini, G. Cicala, UV photocathodes based on nanodiamond particles: Effect of carbon hybridization on the efficiency, Diam. Relat. Mater. 76 (2017) 1. 